\title{
IoT-based Techniques for Online M2M-Interactive Itemised Data Registration and Offline Information Traceability in a Digital Manufacturing System
}

DOI:

10.1109/TII.2017.2704613

\section{Document Version}

Accepted author manuscript

Link to publication record in Manchester Research Explorer

Citation for published version (APA):

Wu, Z., Meng, Z., \& Gray, J. (2017). loT-based Techniques for Online M2M-Interactive Itemised Data Registration and Offline Information Traceability in a Digital Manufacturing System. IEEE Transactions on Industrial Informatics, 13(5), 2397 - 2405. https://doi.org/10.1109/TII.2017.2704613

\section{Published in:}

IEEE Transactions on Industrial Informatics

\section{Citing this paper}

Please note that where the full-text provided on Manchester Research Explorer is the Author Accepted Manuscript or Proof version this may differ from the final Published version. If citing, it is advised that you check and use the publisher's definitive version.

\section{General rights}

Copyright and moral rights for the publications made accessible in the Research Explorer are retained by the authors and/or other copyright owners and it is a condition of accessing publications that users recognise and abide by the legal requirements associated with these rights.

\section{Takedown policy}

If you believe that this document breaches copyright please refer to the University of Manchester's Takedown Procedures [http://man.ac.uk/04Y6Bo] or contact uml.scholarlycommunications@manchester.ac.uk providing relevant details, so we can investigate your claim.

\section{OPEN ACCESS}




\title{
IoT-based Techniques for Online M2M-Interactive Itemised Data Registration and Offline Information Traceability in a Digital Manufacturing System
}

\author{
Zhipeng Wu, Senior Member, IEEE, Zhaozong Meng, Member, IEEE, and John Gray
}

\begin{abstract}
The integration of Internet of Things (IoT) technologies in the industry benefits digital manufacturing applications by allowing ubiquitous interaction and collaborative automation between machines. Online data collection and data interaction are critical for real-time decision making and machine collaborations. However, due to the specificity of digital manufacturing applications, the technical gap between IoT techniques and practical machine operation could hinder the efficient data interactions, collaborations between machines, and the effectiveness as well as accuracy of itemised data collection. This investigation therefore identifies some major technical problems and challenges that current IoT-based digital manufacturing is facing, and proposes a method to bridge the technical gap for itemised product management. The highlights of this investigation are: (1) a data-oriented system architecture toward flexible data interaction between machines, (2) a customised Machine-toMachine (M2M) protocol for machine discovery, presence, and messaging, (3) flexible data structure and data presentation for interoperability, and (4) versatile information tracing approaches for product management. The proposed solutions have been implemented in PicknPack digital food manufacturing line, and achieved ubiquitous data interaction, online data collection, and versatile product information tracing methods have shown the feasibility and significance of the presented methods.
\end{abstract}

Index Terms-Digital manufacturing, M2M, data collection, data interoperability, collaborative automation

\section{INTRODUCTION}

$\mathrm{T}$ HE advances of IoT technologies have created great opportunities and fostered plenty of promising applications for the manufacturing industry [1][2]. The integration of IoT with industry has been a prospective field, where new computing paradigms and business concepts are generated such as Industrial IoT (IIoT), Industry 4.0, smart manufacturing, digital manufacturing [3]. These new computing paradigms highlight the machine connection and collaboration towards a higher level of automation and intelligent decision making in order to introduce more added values and reduce human interventions [4]. The feasibility and readiness of IoT techniques for manufacturing have been discussed in the literature, and it is considered as a new drive to boost the manufacturing industry [5]. Although the vision of digitising

Manuscript received October 04, 2016; revised February 06, 2017; accepted April 27, 2017. This research was supported by the European Commission through PicknPack project (project code: 311987). Corresponding author: Zhaozong Meng (zhaozong.meng@outlook.com).

Copyright (c) 2009 IEEE. Personal use of this material is permitted. However, permission to use this material for any other purposes must be obtained from the IEEE by sending a request to pubs-permissions@ieee.org. manufacturing has gained much interest, it is still relatively unclear how this vision can actually be implemented with concrete standards and technologies. In the technical revolution from traditional manufacturing to digital manufacturing, the high quality connectivity of machines, M2M communication and understanding, data collection and exploration are the major building blocks, while online real-time data collection and data interaction are the fundamentals for collaborative automation, intelligent decision making, and some further merits of IoT-enabled industrial applications.

Information tracking and tracing is mandatory for some industries, such as the food and feed industry [6]. The functionality of itemised data collection, information tracing and process optimisation in digital manufacturing has gone beyond the conventional observation or monitoring process, which includes: (1) identification of individual items such as packages, products, and components, (2) online collaborative operations and decision making between machines, (3) data analysis for industrial process optimisation, and (4) product information traceability for quality control and other purposes.

The novel functionality of collaborative digital manufacturing systems equipped with various sensors and smart devices have created opportunities for itemised data collection and information traceability. Product information traceability systems are conventionally developed to collect online and offline product data for information tracing with respect to product quality or other purposes. There have been studies in both academia and industry about the industrial IoT technologies, such as machine connectivity, communication standards, service architectures, and business models [7][8][9]. However, itemised data collection and management in digital manufacturing under a collaborative automated production environment has not been sufficiently addressed, where identification of product items by all machines through the production process is a key requirement and great challenge.

This investigation aims to identify the related specificities of digital manufacturing application scenarios and propose the corresponding technical solutions for the underlying implementation of online itemised data collection and information traceability. The main features of this investigation are product itemisation in a multi-item manufacturing environment and the corresponding system structure, flexible M2M protocol, interoperable data interaction, and versatile information tracing approaches for product management. A special consideration is made to the proposed methods for the implementation in a food manufacturing line funded by the European Commission under the PicknPack research programme. The hardware and software functionality have 
been developed based on the PicknPack food manufacturing processes. The advantages of the presented solution are demonstrated by the implementation of PicknPack food manufacturing line.

The remainder of this paper is structured as follows: Section II introduces the enabling IoT technologies and related studies in the field of digital manufacturing. Section III illustrates the functionality of PicknPack food manufacturing system and the modular design for collaborative autonomous manufacturing. Then, Section IV discusses the system design and implementation of the proposed solutions. Section V provides the itemised data collection and information traceability of the system with the PicknPack platform. Finally, discussions are given in Section VI and conclusions are drawn in Section VII.

\section{OVERVIEW OF ENABLING IOT TECHNOLOGIES AND APPLICATION INVESTIGATIONS}

By regarding data as a crucial element leveraging the promising functionalities, the relevant supporting techniques can be categorised by their roles in handling data. Based on the categorisation, this section gives an overview of the main building technologies, recent technical progress, and related investigations of IoT technologies for digital manufacturing.

\section{A. Enabling Technologies}

The architecture of IoT can be divided into three layers: perception layer, network layer, and application layer [1]. Since data based machine collaboration and process optimisation are major concerns of smart manufacturing IoT systems, this investigation takes a product as a base item and divides the Industrial IoT system into four layers: machine layer, network layer, service and application layer, and data analytics and optimisation layer, as shown in Fig. 1.

Since sensors and actuators are integrated with industrial modules and are also application specific, we focus on the IoT techniques that build up the digital manufacturing system, which can be categorised into communication level techniques, service and application level techniques, and data and knowledge techniques.

1. Communication Level Techniques

The machine connectivity, presence, discovery, and messaging are the building blocks for M2M communication and machine collaboration in digital manufacturing systems. The M2M communication solutions are being standardised, and many Standard Development Organisations (SDOs) are working toward M2M standards for industry applications, such

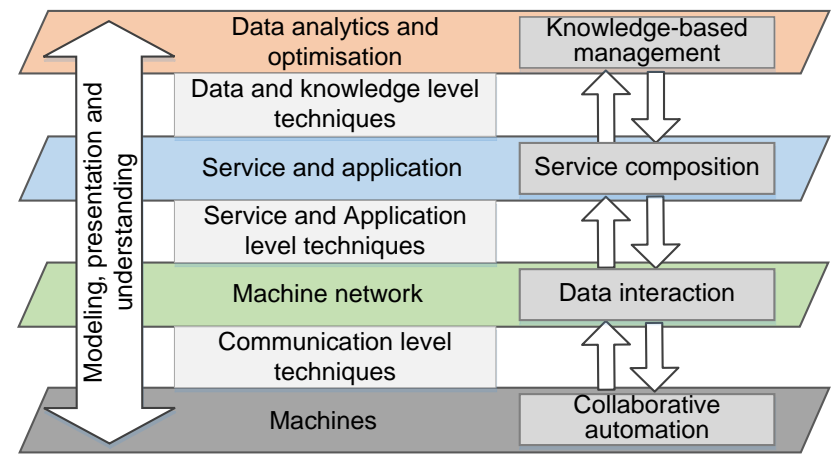

Fig. 1. Categorisation of the Key Enabling Techniques as IEEE, European Telecommunication Standards Institute (ETSI), Internet Engineering Task Force (IETF), $3^{\text {rd }}$ Generation Partnership Project (3GPP), etc. In terms of M2M for industrial scenarios, ETSI M2M service architecture is the first step towards a universal M2M platform, which has already provided a good maturity level on unified communication capabilities and protocols [10]. Many technological solutions and standards have been invented for application specific context, such as WiFi/LTE, WirelessHART, ISA100.11a, etc. [7][11] For interactive machine communications, many optional protocols, middleware, and API libraries are promising for the purpose of M2M messaging, such as Message Queue Telemetry Transport (MQTT), Constrained Application Protocol (CoAP), Data Distribution Service (DSS), Extensible Messaging and Presence Protocol (XMPP), Advanced Messaging Queue Protocol (AMQP), ZeroMQ (ZMQ) [2][12][13]. The M2M communication technologies are invented for different requirements and application scenarios.

2. Service and Application Level Techniques

Application and service level techniques implement the functionality of the system and individual functional modules towards autonomous operations and production strategies.

(1) Data interoperability and semantic understanding

Data presentation and message understanding are the first step of machine collaboration, which potentially allows the machines to understand, share, and reason with semantic technologies at execution time. For data presentation, Extensible Markup Language (XML) and Javascript Object Notation (JSON) are widely used for the representation of arbitrary data structures for Internet computing. Since ontology can be used to establish the relations between variable for some computation for a particular domain of discourse, it is considered a promising solution to organise information and limit complexity in the fields like semantic web, system engineering, and software engineering. Due to the fundamental importance, ontology is widely used in IoT-based systems including digital manufacturing, such as IoT-O to promote the semantic interoperability in oneM2M architecture [14], and Semantic I4.0 Component for Industry 4.0 scenarios [15]. The new method for managing dynamic ontologies in the manufacturing domain can potentially promote the manufacturing enterprise as a highly dynamic environment.

(2) Manufacturing system architecture

The industrial applications of digital manufacturing need not only to handle the data transmission and data interactions but also to support applications for different industrial operations and business purposes. Due to the importance of service architecture in the flexibility and adaptability of production, the mainstream and new emerging computing paradigms are introduced such as RESTful, Service Oriented Architecture (SOA), Resource Oriented Architecture (ROA), Cloud Computing[16]. System architectures are expected to follow a manufacturing-marketplace philosophy that brings together developers, suppliers, and potential customers to collaborate, participate, and produce more efficiently.

There are enterprise or standardised solutions for digital manufacturing, such as Discrete Manufacturing Reference Architecture (DiRA), Smart Manufacturing Leadership Coalition (SMLC) open manufacturing platform, ESTI M2M 
service architecture, oneM2M Global Initiative harmonized reference architecture, etc. [17][18] In addition, customdesigned system architectures are also widely studied. A Manufacturing Reference Architecture (MRA) is proposed in [3], which leverages SOA across integrated manufacturing operations to provide added flexibility. Motivated by the adaptability and responsiveness of manufacturing technologies, ontology, and multi-agent technologies are integrated to introduce united information processing and end-to-end control of manufacturing process in Adaptive Production Management (ARUM) approach [19], which can promote the adaptability and on-the-fly responsiveness in production management. In order to support different applications for business purposes, a configurable information service platform is proposed in [20]. By combining ontology and RESTful service, the platform provides an information support base for data integration and intelligent interaction toward product lifecycle management.

In summary, the architectures are designed to promote the flexibility of manufacturing ranging from information composition, transferring and interaction to post-production business proposes. Since the underlying heterogeneity in hardware, software and data, interoperability plays a very important role to deal with the potential technical challenges [21]. Also, due to the heterogeneity in manufacturing systems and manufacturing strategies, there is still no widely accepted system architecture.

3. Data and Knowledge Techniques

As mentioned above, data is the most important factor in a manufacturing system allowing machine collaboration and intelligent optimisation. Knowledge Discovery in Database (KDD) and big data analysis are the methods to exploit the itemised data for further optimisation of machine configurations, industrial processes, or production strategies, etc. Techniques of this level link the production system to source materials and retailing market in an integral business model, which can conversely optimise the production system. Data and knowledge level techniques are also expected to introduce intelligent functionalities including novel database technology, data analytics, and knowledge-based optimisation.

The analysis of itemised product data can be potentially used to enable dynamic controlled collaboration through manufacturing. The manufacturing blueprint which composes, integrates, and contextualises manufacturing processes and data can be implemented in a knowledge structure for dynamic optimisation. However, it is a challenge to transform information from a vast array of diverse sources into useful knowledge to support effective decisions and planning. Some machine learning and data mining tools for data analysis are needed to implement and achieve the analytic capabilities. Data analysis and knowledge-based management will bring more benefits to the manufacturing industry.

\section{B. Opportunities and Challenges}

From the overview above, the integration of information techniques can created chances to innovate the manufacturing industry. However, the heterogeneous techniques of different levels raise challenges in data interoperability. To bridge the gap between techniques of different realms is a critical fundamental task for digital manufacturing systems. To fix the problems, it is noteworthy to consider the characteristics that distinguish digital manufacturing applications from other IoT scenarios, which can be summarized as follows:

(1) Powerful machines and reliable network connection

(2) Machines make decisions and fulfill operations locally

(3) Machines generate data, and consume data from peers

(4) Interactive and high throughput data communication

These features make industrial manufacturing applications different from light-weight and centralized monitoring IoT applications. It requires higher performance in inter-module connectivity, discovery, and messaging between machines. With respect to data collection and data exploration, data interoperation and data integration are challenging tasks because of heterogeneity in devices and technologies. On one hand, the systematic design of data structuring, cross-platform interoperation, and semantic understanding are critical issues for data interoperation and collaborative operations between the machines. On the other hand, each functional module, either as a data generator or a data consumer needs to identify IDs of the products on the dynamic manufacturing line, and at the same time collects and integrates data from them for further uses.

As the functionalities of a manufacturing system are application specific, this investigation focuses on the design principles and critical underlying enabling techniques, such as product identification, online data collection and data integration methods that suit the digital manufacturing scenarios with the production of multiple items simultaneously on a single line. As an example, the methods are implemented in the PicknPack food manufacturing line which is described in the following sections.

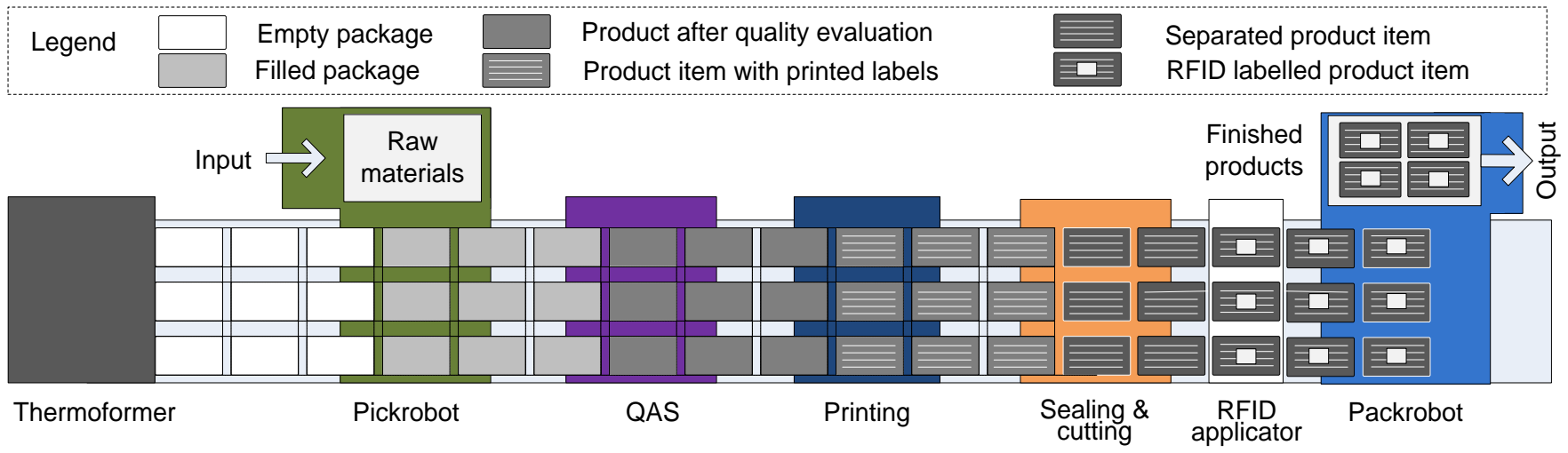

Fig. 2. Schematic Diagram of PicknPack Manufacturing Line 


\section{PicknPack Digital FoOd ManUfacturing Line}

The PicknPack research programme is a timely practice of Industrial IoT, smart manufacturing and digital manufacturing concepts when they are proposed. It aims to integrate the stateof-the-art robotics, sensors, and controllers in the industry with emerging information and communication technologies to build a flexible automatic food manufacturing line. The concept is to connect each functional module within a network, and the modules perform their functionalities in real-time collaboration with peer modules within the system in a decentralised way. This decentralised structure allows more flexible M2M messaging between machines for decision making and collaborative operations.

The food production process of PicknPack includes the following steps: product package fabrication, automatic source food filling, quality assessment and sensing (QAS), dynamic label printing, laser sealing and cutting, product packaging, and product information tracking and tracing. Fig. 2 gives the schematic diagram of the hardware system. The physical functional modules in this system are:

- Thermoformer - A machine that produces empty food packages in a row simultaneously from a flat plastic film, with each package registered with a Universal Unique Identifier (UUID).

- Pickrobot - A robot that picks raw food substances from an input crate, and places them into the newly made packages forming individual product items, as the packages reach its workspace.

- QAS - A sensing system that evaluates the quality of food with RGB, 3D, hyperspectral, microwave and X-ray sensors, as the packages reach its workspace.

- Printing - A machine that prints the required information and QR code on each product item, as the packages in its workspace.

- Sealing \& Cutting - A laser system that seals labels on packages and cuts them into separate product items in its workspace.

- Packrobot - A robot that picks up the separate product items and put them into an output crate, as they reach its workspace.

- Traceability module - A RFID based software module that monitors the production operation, collects data and saves them to database for further use, while an RFID applicator is used to place RFID labels to individual product items.

- Line controller - A module that manages the configuration of the peer modules and coordinates their interactions.

As multiple empty food packages are produced simultaneously in a row, the virtual UUID scheme is proposed to register the packages as well as the product items at the manufacturing stage. This UUID can be linked to RFID for data integration and product information traceability. In the food manufacturing, the food packages move in rows and columns along the line, from empty packages to filled product items.

The above individual modules work collaboratively to complete the automatic food manufacturing process. Some modules need to collaborate with peer modules in order to accomplish their tasks. For instance, the printing module needs to have the UUID of each product item as well as weight, pricing policy, supplier and quality information from Pickrobot, QAS and other modules to print on the labels. To achieve the automatic functions, all functional modules are connected to the local area network and operate in an automatic way.

The following sections detail the technologies underpinning the success of the system focusing on data communication, data interaction, messaging, presentation and understanding, and collaborative automation of machines.

\section{System ARchitecture DESIGN FOR PICKNPACK DIGITAL FOOD MANUFACTURING LINE}

Regarding data interoperability as a key parameter to enable digital manufacturing applications, this section provides a dataoriented system architecture to allow data interaction between machines and online unobtrusive data collection with each package as a base item. The corresponding technical designs are illustrated to bridge the gap between heterogeneous techniques and achieve interoperable data interaction and collaborative automation in the PicknPack manufacturing line.

\section{A. System Architecture Design}

It is the instant communication between machines that allows the flexible industrial automations and further data based intelligence. Since machines in the system carry out their specific operations with the assistance of peer machines, the individual operational machines need to be organised in an architecture with flexible connectivity and open data interfaces. The proposed data-oriented system architecture below presents the hierarchy of functionality, connectivity of machines, M2M messaging and understanding, and inter-module data communications with consideration of itemised information registration.

As mentioned above, the UUID is used to distinguish the product items. To denote the function of machine $i$ with $M_{i}$ and output data of machine $i$ is $D_{i}$, the output data collection of product $D(U U I D)$ can be denoted with:

$$
D(U U I D)=\sum_{i=1}^{n} D_{i}(U U I D)=\sum_{i=1}^{n} M_{i}(U U I D)
$$

Evidently, it is a critical task for each machine to identify the UUID of the product items and integrate the corresponding data it generates with UUID

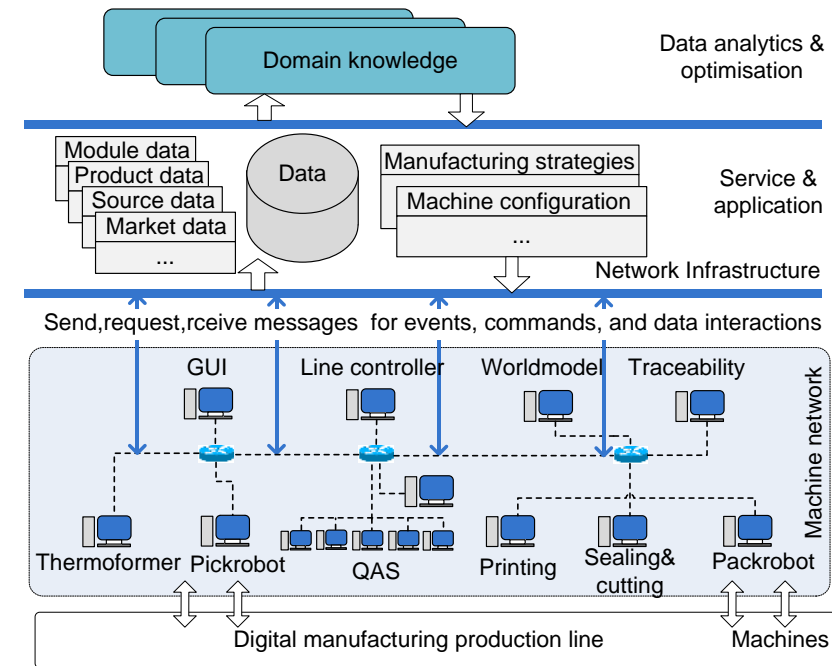

Fig. 3. Data-Oriented System Architecture 
Evidently, it is a critical task for each machine to identify the UUID of the product items and integrate the corresponding data it generates with UUID.

1. Data-Oriented System Architecture Design

According to the hierarchical structure of the enabling techniques for industrial IoT systems, a data-oriented system architecture is designed as shown in Fig. 3. The machines are enabled with M2M communication protocols which can talk to each other for collaborative operations. The service and application-level functionalities specify the machine operations, event handling models, machine configuration, and production strategies. Data and knowledge level techniques take advantage of the online collected data and domain knowledge to supervise the industrial manufacturing operations. The data interaction and data interoperability are regarded as central issues because all critical collaborations are based on the data interaction and understanding, such as event notification and joint decision making.

2. Machine Connectivity and Machine Topology

Different from some sensor monitoring applications, the amount of data of the messages during production may reach hundreds $\mathrm{K}$ bytes/second or $\mathrm{M}$ bytes/second and the types of data in communication may also include images. On the other hand, the interactions are expected to be handled in real-time. The connectivity between machines can be based on reliable wired/wireless connections. Networking solutions for resourcelimited machines or non-reliable network are not considered.

To build an effective M2M messaging connectivity between machines, the roles of machines in the manufacturing system are categorised into three levels: line, module, and device.

- Line - The top level that includes all modules and devices that constitute the manufacturing system.

- Module - Module means a group of devices that fulfill some complete functions. One module may contain one device, or multiple devices with a module controller.

- Device - Device is the minimum element in the system that completes a particular function of the manufacturing line.

The connectivity of machines is as shown in Fig. 3. All module controllers and devices are connected to a local area network. Each machine can talk to any other machines following an M2M communication protocol.

\section{B. M2M Communication and Machine Collaboration}

It is evident that all the functions are achieved based on the M2M communication mechanism and semantic understanding between machines, which allows the data interactions, event notifications and responses to be triggered correctly in realtime. Therefore, the connected machines need to be integrated with enabling techniques for flexible communication and understanding of messages, namely the M2M messaging protocol, data modelling and presentation.

\section{M2M Messaging Protocol}

Due to the aforementioned features of digital manufacturing applications in non-lightweight, highly interactive, real-time messaging, etc., Zyre - a ZMQ based open source framework is chosen for machine connectivity and the implementation of the desired M2M messaging functions. The ZMQ Real-time Exchange (ZRE) protocol manages how a sub-group of peers on a network discover each other, organise into groups, and

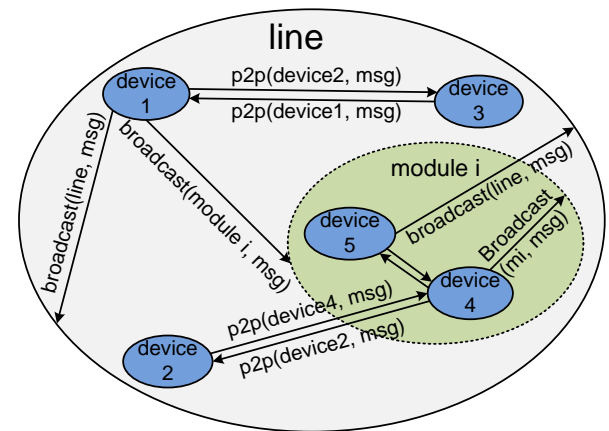

Fig. 4. M2M Messaging between Machines

send each other events. ZRE runs over the ZMQ Message Transfer Protocol (ZMTP). With Zyre, machines can talk to one another and one-to-multiple of grouped machines. Machines must be able to know all the connected peers available in the network and their states of connection. A unique device name and Unique Machine Identifier (UMID) for each machine are created to identify the machines in the network. The heartbeating mechanism allows the machines to notify their state to the peers and be notified the peers' states as well.

Zyre uses IPv4 beacon broadcasts to discover machines. Each machine shall listen to the Zyre discovery service at a particular UDP port. Each machine shall broadcast, at regular intervals, on the UDP port a beacon that identifies itself to any listening machines in the network. In this case, each machine can maintain a lookup table consisting of the information of all connected machines. The machines keep their local lookup table updated by giving notification of event such as machine joining and leaving through beacon messages. In this way, machines make themselves known when joining the network and also discover information of all existing machines, and connected machines keep updated of the states of all peer machines.

Enabled by the machine discovery and heartbeating techniques, a machine can identify behaviours of the peer machines such as entering the network, joining or leaving a group, and exiting the network. The machines can initiate or receive $\mathrm{P} 2 \mathrm{P}$ communication and broadcast through software functions 'whisper' and 'shout' respectively.

As shown in Fig. 4, in the overall group 'line', there are devices and modules as sub-groups. A device machine can talk to any device in the overall group using 'whisper'. A device can also broadcast to any sub-group so that all the machines in the sub-group can receive the message. This versatile messaging function makes data transfer and event notification between machines very flexible.

\section{Machine Understanding}

To strengthen the understanding between machines, an ontology is designed to model the entities, parameters, states, commands, and events of the product manufacturing. All machines access the models and configurations when starting the system, and then initiate the machines, process the data, trigger the events, and carry out the manufacturing operations.

For data presentation, JSON is applied and a reference messaging format is given in Fig. 5. All the machines need to follow this format to generate messages and parse received messages. The top level nodes give the information of the origin of the message, the destination of the message, the type of message and the time stamp. Then in the extended node 


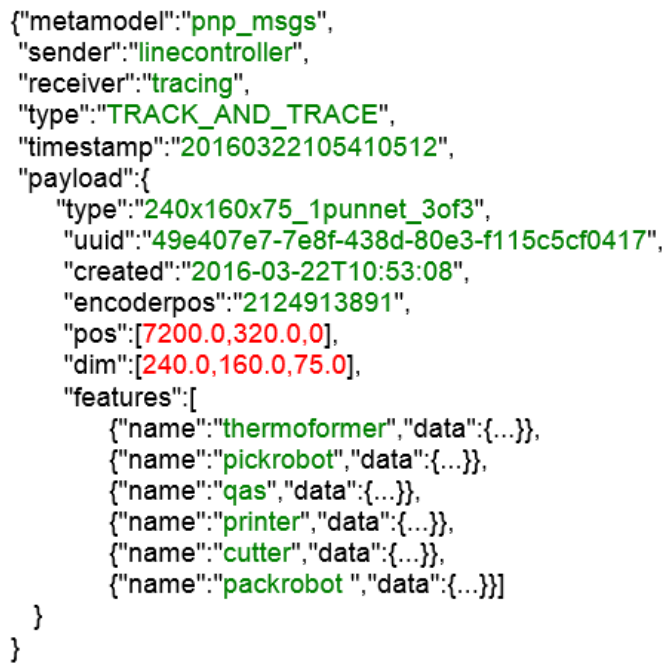

Fig. 5. A Reference Message in JSON

'payload', structured information can be enclosed, such as product features and the configuration of a machine. The example message in Fig. 5 is the linecontroller which temporary save product information sending product features to the traceability module. Message for other M2M communication may have a node other than 'features' in 'payload'.

Since JSON is a cross-platform method for data presentation over a network, the events, commands, and data can be structured and wrapped with JSON for data transfer between machines. The receiving machine can parse the received message and get the useful parameters for calculation and decision making.

3. Collaborative Automation

The instant messaging and understanding between machines leverages online intelligent decision making by accessing relevant data from the peer modules. Therefore, the industrial operations can be fulfilled in a collaborative way. The machine collaboration is illustrated with the following two examples:

Example 1: Dynamic label printing

As shown in Fig. 6, the printing module can obtain the UUIDs of the product items according to the encoder position from Thermoformer, access quality information collected by the QAS module and source information collected by Pickrobot, and print them on the labels. Therefore, the data of a specific product item can be accessed and printed on its label in realtime.

Example 2: Online product quality classification

As shown in Fig. 7, when the Packrobot picks up a product item, it can obtain the UUID of the product item, access source material data and food quality information, and check the correctness of printing as well as sealing and cutting quality.

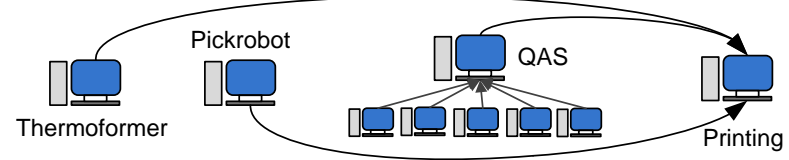

Fig. 6. Dynamic Label Printing

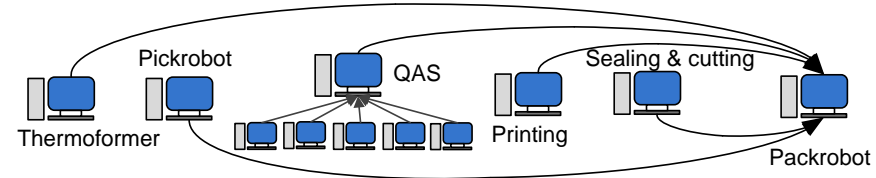

Fig. 7. Online Product Quality Classification
Based on the information, the Packrobot can then sort its quality and put it into the crates of different quality classes or reject it.

The collaborative automation of machines can largely reduce human interventions in the manufacturing process. The flexible inter-module communication also enables the observation of the whole industrial application and allows the traceability module to collect data from all functional machines.

\section{Synchronisation of Industrial Operations}

In order to correctly perform the automatic manufacturing process and collect the corresponding data, the functional modules in the whole manufacturing line must be synchronised. That is to say, all machines need to know the UUID of products, carry out the particular operation at the correct time, and link the data to the UUID. For some devices like the sensor devices, they need to know the exact physical start and end positions of the package object as it moves in order to scan and get the quality parameters. Implementation of the synchronisation and data integration requires both hardware and software collaboration. This system utilises an accurate mechanical encoder installed in the Thermoformer which broadcasts the UUIDs and encoder positions of the packages to all other machines as they are created. Other machines register the package positions in their workspaces, carry out their operations, and assign the corresponding data to the packages identified by UUIDs.

With the presented system architecture, flexible machine communication, machine understanding, and data integration methods, the collaborative automation and intelligent optimisation are thus made possible. In order to make the messaging protocol applicable to all machines in the system, software interfaces are developed to integrate with different platforms such as LabVIEW, MS C\#.NET, and C++. For some proprietary machines, a controller $\mathrm{PC}$ is used as an intermediary to translate the protocols and manage the data interactions.

\section{RFID BASED PRODUCT INFORMATION TRACEABILITY}

The product information traceability system is a separate module in the PicknPack food manufacturing line. Its task is to collect the online and offline data, integrate the data, and provide technical methods for information tracing and analysis. The system collects online generated data based on the machine collaboration with the designed system architecture. The design of product data collection and tracing functions and the technical solutions are illustrated in this section.

The online information is in connection with UUID, which can be used locally for the machines to identify the product items. However, when the products are offline, it is difficult to identify them and access the corresponding information. To deal with this problem, RFID and QR code are the ideal candidates. With the RFID readers and antennas installed on the manufacturing line, the system can monitor the RFIDs of input crate, product items, and output crate. Since each product item has a UUID and RFID, the online and offline information can be integrated, and traced back with RFID system conveniently.

The design of the RFID traceability system is as shown in Fig. 8, one RFID antenna is installed in the Pickrobot to monitor the input crates, one is installed with RFID applicator to link UUID with product item RFID, and the other one is installed in 
Packrobot to monitor the output crates. The detailed technical designs are illustrated as follows.

\section{A. Data Modelling and Data Integrity}

Among the RFIDs used, the input crate is linked to source material information, the product item RFID binding with UUID links with product information created by the modules in the manufacturing line, and the output crate RFID links with

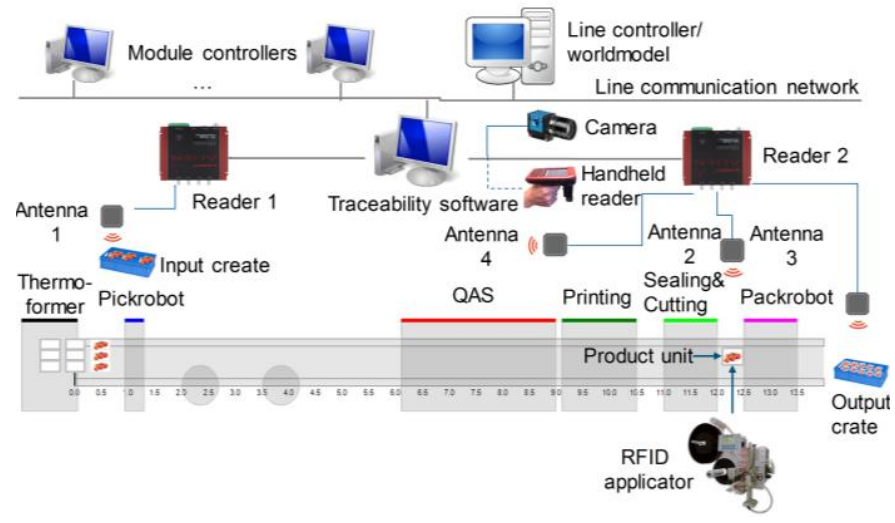

Fig. 8. RFID based Product Information Traceability System logistical and market information. Each product item can be identified with UUID, RFID, QR code, while the input crate and output crate are identified with RFIDs. The relationship of the IDs and information can be presented the diagram in Fig. 9.

A custom designed database is developed according to the product data model for the traceability function. The traceability module software accesses all machine configurations, online generated data, offline registered data, and business partner provided data in the database.

\section{B. RFID Product Identification for Process Monitoring}

A critical step to integrate all information of a product item is to link the product item RFID to the corresponding UUID. This is achieved by the traceability software and RFID applicator. The traceability system as a virtual module receives encoder position, and identifies the UUID of each package. An RFID is then placed on the package when it reaches the workspace of the RFID applicator. The RFID identified by RFID reader, which is placed on the product item, will be linked to the UUID of package. To write the UUIDs in the RFID chips is also a feasible technical solution, which simplifies the database structure and software application.

With the input crate RFID, product item RFID, and output crate RFID as well as UUID, the information management in the manufacturing operation can be carried out automatically. By linking the relevant IDs, all related information can be integrated to a product item, which is useful for further analysis and the optimisation of the manufacturing system.

The RFID technology allows the instant product information tracing, which is useful for many industrial applications. The UUID is also encoded with a QR code and printed on the product labels, which provides another convenient way for itemised product information tracing.

\section{Versatile Product Information Tracing Approaches}

Data visualisation is a useful function for the information traceability system. It is also expected to carry out the data traceability process conveniently. Different from the traditional

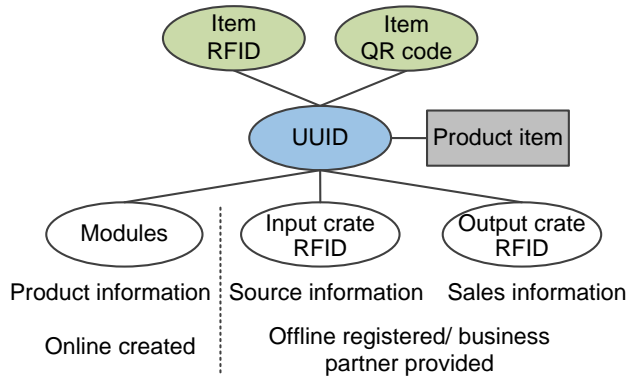

Fig. 9. Product Information Data Modelling

manual information tracing, the PicknPack traceability system allows three traceability methods: RFID, QR Code and UUID.

- RFID tracing - by placing RFID labeled product items close to RFID antenna to access the product information.

- QR Code tracing - by placing the product items with printed QR code to a camera to access the product information.

- UUID tracing - By inputting or selecting a UUID from a list to access the product information with the traceability software.

The application of RFID has facilitated both the production process and offline product information tracing. The traceability system is a useful tool for quality control and system optimisation. The information to trace in this system is not limited to text data. Images are also saved in some devices for performance analysis for the modules and the line.

\section{KDD and Optimisation Model}

The significance of the digitalisation for a manufacturing system can be summarised into three aspects: (1) provision of higher degree of automation, (2) comprehensive collection and registration of parameters of production and products, and (3) enabling system optimisation based on data analysis. Therefore, in addition to product information tracing, the exploration of collected data for system optimisation is an important function that brings benefits to the production. For the PicknPack production line, some statistic parameters can be obtained with the collected data, such as quality class ration, rejection rate, and sales volume with different production strategies and source materials. The parameters can be used to evaluate the performance of the modules and devices, the efficiency of the production policy, and effectiveness of machine configurations etc.

Some machine learning mathematical models such as Bayesian Network, K-Nearest Neighbours (KNN), Support Vector Machine (SVM) and Artificial Neural Network can be used to classify the collected data and determine the underlying rules. The potential functionality of system optimisation can help operators to devise suitable production strategy and evaluate the performance of functional modules, devices and the line as a whole. Problems like machine failure and incorrect initiation that affects the production can be identified by the data analysis application. Exploration of the collected data can potentially allow the system to improve the performance conveniently, which was not possible for traditional systems.

\section{DISCUSSION}

Digital manufacturing is a multi-disciplinary field, and many related investigations view it from an information science perspective. However, the practical technical challenges facing 
TABLE 1. Key Parameters, Technologies, and Achievements

\begin{tabular}{|c|c|c|}
\hline Parameters & Technologies & Functions Achieved \\
\hline $\begin{array}{l}\text { Connection, } \\
\text { presence, } \\
\text { discovery }\end{array}$ & Heartbeating & $\begin{array}{l}\text { Easy creation of connection and flexible } \\
\text { entry, leaving, discovery and presence } \\
\text { Easy to organise and maintain }\end{array}$ \\
\hline $\begin{array}{l}\text { Ubiquitous } \\
\text { data access }\end{array}$ & $\begin{array}{l}\text { Zyre-based M2M } \\
\text { messaging }\end{array}$ & $\begin{array}{l}\text { Flexible messaging for events } \\
\text { notification and data exchanging } \\
\text { Supporting collaborative operations }\end{array}$ \\
\hline $\begin{array}{l}\text { Interoperable } \\
\text { understanding }\end{array}$ & $\begin{array}{l}\text { Ontology } \\
\text { modelling, JSON } \\
\text { presentation }\end{array}$ & $\begin{array}{l}\text { Support of cross-platform machine } \\
\text { interoperability }\end{array}$ \\
\hline $\begin{array}{l}\text { Data } \\
\text { integration }\end{array}$ & $\begin{array}{l}\text { Encoder position, } \\
\text { UUID, and RFID }\end{array}$ & $\begin{array}{l}\text { Integration of online generated and } \\
\text { offline registered data } \\
\text { Synchronisation of virtual model with } \\
\text { physical operations }\end{array}$ \\
\hline $\begin{array}{l}\text { Flexible } \\
\text { manufacturing }\end{array}$ & Overall modelling & $\begin{array}{l}\text { Easy configuration and reconfiguration } \\
\text { of overall system }\end{array}$ \\
\hline
\end{tabular}

the industrial applications have not been sufficiently addressed. This investigation thus focuses on the online product identification and data interaction between machines for itemised product data management and automatic manufacturing, and provides technical solutions for machine discovery, messaging, data presentation and understanding to enable collaborative automation between machines. It highlights the specificity of digital manufacturing systems that distinguish from light-weight IoT applications in machine computation power, decentralised computing, and interactive communication, and provides M2M communication protocol and data presentation solution for inter-module communication and understanding between machines. The flexible machine messaging and understanding therefore allow online real-time decision making and collaborative automation of machines.

Another technical issue which is seldom addressed is the real-time synchronisation of virtual models and physical operations and the data integration based on the synchronisation. A mechanical encoder based line synchronisation is implemented so that all modules can use the UUID of product items for data integration. This resolves an indispensable technical issue to enable digital manufacturing.

In addition, the decentralised machine communication architecture allows ubiquitous data access between the machines. Compared to centralised structure, its strengths are: (1) more flexible and efficient M2M interactions; (2) no communication and computation bottleneck caused by a central machine; and (3) easy maintenance and convenient upgrading.

With the proposed solution, the practical application of the PicknPack line has overcome some technical challenges and achieved the main manufacturing functionalities that allow the flexible organisation and collaboration of machines. The major parameters, technologies applied, and functions achieved are summarized in TABLE 1.

\section{CONCLUSION}

The data interaction techniques presented in this paper allows comprehensive monitoring of the information regarding the manufacturing operations. It promises the flexibility of production across the entire manufacturing and supply chains, and allows complete product lifecycle management between business partners. The application in the PicknPack line has proved the design concept presented in this paper.

Firstly, the presented system architecture highlights the dataoriented design principle and outlines the main enabling technologies for industrial manufacturing application scenarios, particularly with itemised data registration and management. The M2M communication protocol and machine topology allow flexible messaging between all connected machines, and the interoperable data presentation method leverages crossplatform understanding of the messages. The collaborative automation between machines results in a higher level automation of the manufacturing operation.

Secondly, the line synchronisation and data integration method are the highlights of this work. With the encoder position information a module can register the product UUID and identify the product when it is in the workspace of the module. The data generated by the module can be collected by the production system for automatic operations, which also creates opportunities for further intelligent optimisation.

The RFID technology provides a way to access the online generated information of products. By monitoring the input container and output container with RFID, the offline registered information such as source material information, and customer and delivery information are integrated with the online generated information. The convenient product information tracing with RFID and QR code makes the data available to all partners in the supply chain and even end users.

The work presented in this paper focuses mainly on the communication and application level techniques for digital manufacturing applications. The corresponding designs enable itemised data collection, data interaction, and collaborative operations. However, the further exploration of the collected data is the solution to create intelligence in manufacturing and therefore improve the performance of the whole system. The study of data analysis method is a focus of the future work. Not limited to the manufacturing line level, the system optimisation can be extended to enterprise level with a business model of the manufacturing line, previous and next business partners, etc. The data science technologies has created very wide space for the comprehensive optimisation of industrial applications.

\section{REFERENCES}

[1] Q. Chi, H. Yan, C. Zhang, Z. Pang, and L. Da Xu, "A reconfigurable smart sensor interface for industrial WSN in IoT environment," IEEE Trans. Ind. Informatics, vol. 10, no. 2, pp. 1417-1425, 2014.

[2] L. Xu, W. He, and S. Li, "Internet of Things in Industries: A Survey," IEEE Trans. Ind. Informatics, vol. 10, no. 4, pp. 2233-2242, 2014.

[3] M. P. Papazoglou, W. J. Van Den Heuvel, and J. E. Mascolo, "A reference architecture and knowledge-based structures for smart manufacturing networks," IEEE Softw., vol. 32, no. 3, pp. 61-69, 2015.

[4] J. Davis, T. Edgar, J. Porter, J. Bernaden, and M. Sarli, "Smart manufacturing, manufacturing intelligence and demand-dynamic performance," Comput. Chem. Eng., vol. 47, pp. 145-156, 2012.

[5] Z. Bi, L. Da Xu, and C. Wang, "Internet of things for enterprise systems of modern manufacturing," IEEE Trans. Ind. Informatics, vol. 10, no. 2, pp. 15371546, 2014.

[6] S. Charlebois, B. Sterling, S. Haratifar, and S. K. Naing, "Comparison of Global Food Traceability Regulations and Requirements," Compr. Rev. Food Sci. Food Saf., vol. 13, no. 5, pp. 1104-1123, 2014.

[7] F. Ghavimi and H. H. Chen, "M2M communications in 3GPP LTE/LTE-A networks: Architectures, service requirements, challenges, and applications," IEEE Commun. Surv. Tutorials, vol. 17, no. 2, pp. 525-549, 2015.

[8] K. Xu, Y. Qu, and K. Yang, "A tutorial on the internet of things: From a heterogeneous network integration perspective," IEEE Netw., vol. 30, no. 2, pp. $102-108,2016$.

[9] H. Cai, L. Xu, B. Xu, C. Xie, S. Qin, and L. Jiang, "IOT-based Configurable Information Service Platform for Product Lifecycle Management," IEEE Trans. Ind. Informatics, vol. PP, no. 2, pp. 1-1, 2014.

[10] J. Latvakoski, M. Alaya, H. Ganem, B. Jubeh, A. Iivari, J. Leguay, J. Bosch, and N. Granqvist, Towards Horizontal Architecture for Autonomic M2M Service Networks, vol. 6, no. 2. 2014.

[11] W. Ikram, S. Petersen, P. Orten, and N. Thornhill, "Adaptive Multi-Channel 
Transmission Power Control for Industrial Wireless Instrumentation," IEEE Trans. Ind. Informatics, vol. 3203, no. c, pp. 1-1, 2014.

[12] A. Al-Fuqaha, A. Khreishah, M. Guizani, A. Rayes, and M. Mohammadi, "Toward better horizontal integration among IoT services," IEEE Commun. Mag., vol. 53, no. 9, pp. 72-79, 2015.

[13] P. Hintjens, ZeroMQ: Messaging for Many Applications. Sebastopol, USA: O'Reilly Media, Inc., 2013.

[14] M. Ben Alaya, S. Medjiah, T. Monteil, and K. Drira, "Toward semantic interoperability in oneM2M architecture," IEEE Commun. Mag., vol. 53, no. 12, pp. 35-41, 2015.

[15] I. Grangel-González, L. Halilaj, G. Coskun, S. Auer, D. Collarana, and M. Hofmeister, "Towards a Semantic Administrative Shell for Industry 4.0 Components," arXiv Prepr. arXiv1601.01556, 2016.

[16] S. Savazzi, V. Rampa, and U. Spagnolini, "Wireless Cloud Networks for the Factory of Things: Connectivity Modeling and Layout Design," IEEE Internet Things J., vol. 1, no. 2, pp. 180-195, 2014.

[17] Microsoft Discrete Group Manufacturing, "A Discrete Manufacturing Reference Architecture (DIRA) Framework Whitepaper," 2011.

[18] P. O’Donovan, K. Leahy, K. Bruton, and D. T. J. O'Sullivan, “An industrial big data pipeline for data-driven analytics maintenance applications in large-scale smart manufacturing facilities," J. Big Data, vol. 2, no. 1, p. 25, 2015.

[19] D. T. Meridou, A. P. Kapsalis, M. E. C. Papadopoulou, E. G. Karamanis, C. Z. Patrikakis, I. S. Venieris, and D. T. I. Kaklamani, "An Ontology-Based Smart Production Management System,” IT Prof., vol. 17, no. 6, pp. 36-46, 2015.

[20] J. Swetina, G. Lu, P. Jacobs, F. Ennesser, and J. Song, "Toward a standardized common M2M service layer platform: Introduction to oneM2M," IEEE Wirel. Commun., vol. 21, no. June, pp. 20-26, 2014.

[21] G. Xiao, J. Guo, S. Member, and Z. Gong, "User Interoperability With Heterogeneous IoT Devices Through Transformation," IEEE Trans. Ind. Informatics, vol. 10, no. 2, pp. 1486-1496, 2014.

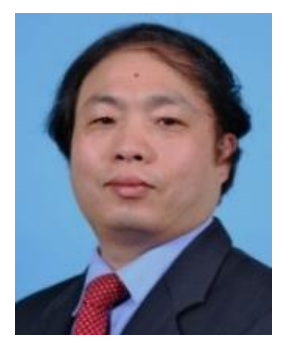

Zhipeng Wu (M'95-SM'05) received the B.Sc. degree in radio technology from Northeast University, Shenyang, China, in 1983, and the Ph.D. degree in antennas and propagation and the D.Eng. degree in antennas and microwave engineering from the University of Birmingham, Birmingham, U.K., in 1988 and 2003, respectively. From 1988 to 1991, he was appointed as a Research Fellow with the University of Birmingham. In 1992, he joined The University of Manchester Institute of Science and Technology, Manchester, as a
Lecturer. He is currently a Professor of Antenna and Propagation with The University of Manchester, Manchester, U.K. His research interests include microwave sensors, RF and microwave tomography, WSNs, Internet of Things, and techniques and systems for industrial applications.

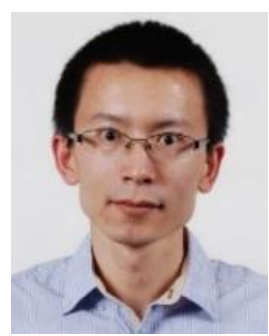

Zhaozong Meng (M'16) received the B.Sc. degree in instrumentation and measurement from Sichuan University, Chengdu, China, in 2006, the M.Sc. degree in instrumentation and measurement from Beihang University, Beijing, China, in 2009, and the Ph.D. degree in computer science from the University of Huddersfield, Huddersfield, U.K., in 2014. He joined The University of Manchester, Manchester, U.K., as a Research Associate, in 2014. His research interests include sensors, instrumentation and measurement, wireless sensor networks, M2M communications, and industrial Internet of Things.

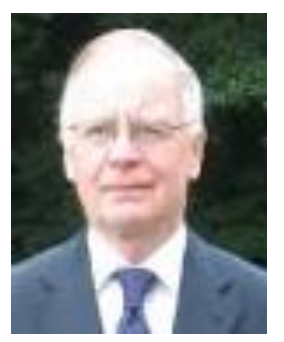

John Gray received his B.Sc. degree in electrical engineering and M.Sc. in semiconductor electronics from Queens University Belfast, Belfast, U.K. in 1959 and 1961 respectively, and the Ph.D. degree in control systems from The University of Manchester, Manchester, U.K. in 1967. He was appointed a Lecturer in electrical engineering with University of Manchester. In 1979, he was appointed as the Chair of Control Engineering, University of Salford, Greater Manchester, U.K. He is currently a Professor in robotics and systems engineering with The University of Manchester. His research interests include robotics, automation in food manufacturing, and digital manufacturing. 